$\left(E C_{50}{ }^{\mathrm{DPPH}}=0.071 \pm 0.003 \mathrm{mg} / \mathrm{ml}\right)$. Both tomato waste extracts influenced cell growth depending on cell line and extract dose. The strongest activity against cancer cells was observed by Bačka extract in HeLa cell line $\left(\mathrm{EC}_{50}{ }^{\mathrm{He}-}\right.$
$\mathrm{La}=0.51 \pm 0.02 \mathrm{mg} / \mathrm{ml}$ ). The obtained results show that tomato waste could represent a cheap and readily available source of value-added ingredient for functional food and some other health products.

\title{
MICROPROPAGATION OF GLOSSOGYNE TENUIFOLIA CASSINI - A TRADITIONAL MEDICINAL PLANT
}

\section{(c) Chang Hung-Chi ${ }^{1,3}$, Kuo Chao-Lin ${ }^{2}$, Karuppaiya Palaniyandi ${ }^{3}$, Tsay Hsin-Sheng ${ }^{3,4}$}

${ }^{1}$ Department of Golden-Ager Industry Management, Chaoyang University of Technology, Taichung, Taiwan ${ }^{2}$ School of Chinese Pharmaceutical Sciences and Chinese Medicine Resources, China Medical University, Taichung, Taiwan ${ }^{3}$ Institute of Biochemical Sciences and Technology, Chaoyang University of Technology, Taichung, Taiwan

${ }^{4}$ Department of Agronomy, National Chung Hsing University, Taichung, Taiwan

Glossogyne tenuifolia Cass. (Hsiang Ju in chinese) belongs to the family Asteraceae and originates in Penghu Island, Taiwan and it is used to make traditional healthy food and drink consumed in Penghu Island.G. tenuifolia has a long history of being use as an antipyretic, hepatoprotective and anti-inflammatory remedy in folk medicine among local residents. Oleanolic acid and luteolin-7-glucoside are main active compound of $G$. tenuifolia. The levels of these active compounds in G. tenuifolia are affected by many factors including climatic change and pathogens. The plant cell culture is a promising potential alternative technique to produce high value sec- ondary metabolites. Therefore, the present study aims to develop a micropropagation protocol for $G$. tenuifolia. Maximum numbers of shoots ( 2.4 shoots/explant) were obtained from in vitro seedling-derived shoot tip cultured on half-strength Murashige \& Skoog (1962) (MS) medium supplemented with $0.1 \mathrm{mg} / \mathrm{L}$ benzyladenine. The well-developed shoots were rooted on half strength MS medium supplemented with $0.5 \mathrm{mg} / \mathrm{L}$ Napthaleneacetic acid and $0.1 \mathrm{mg} / \mathrm{L}$ putrescine. Eighty percent of the inoculated shoots produced roots with an average of 3.4 roots/shoot. The rooted plantlets were successfully acclimatized in greenhouse with $100 \%$ survival rate.

\section{ANTIOXIDANT ACTIVITY OF KOELREUTERIA HENRYI DUMMER FLOWER}

\section{() Chen Jial-Jhen ${ }^{1}$, Chang Hung-Chi ${ }^{2,3}$, Karuppaiya Palaniyandi ${ }^{3}$, Tsay Hsin-Sheng ${ }^{3,4}$}

'School of Chinese Pharmaceutical Sciences and Chinese Medicine Resources, China Medical University, Taichung, Taiwan ${ }^{2}$ Department of Golden-Ager Industry Management, Chaoyang University of Technology, Taichung, Taiwan ${ }^{3}$ Institute of Biochemical Sciences and Technology, Chaoyang University of Technology, Taichung, Taiwan ${ }^{4}$ Department of Agronomy, National Chung Hsing University, Taichung, Taiwan

Koelreuteria henryi Dummer (Known as Golden Flame Tree in Taiwan) is a deciduous tree native to Taiwan belonging to the family Sapindaceae. It has been traditionally used in folk medicine for the treatment of diarrhea, malaria and urethritis. The present study was undertaken to find the antioxidant properties of the flower of Koelreuteria henryi Dummer by DPPH radical-scavenging activity, Trolox equivalent antioxidant activity, and ferric reducing antioxidant power assay and phytochemical analysis. The flowers were extracted with five different polar solvents: distilled deionized water, methanol, ethanol, ethyl acetate and hexane. Maximum yield of antioxidant components were observed in water extract $(27.2 \%)$ followed by ethanol extract $(20.2 \%)$ and methanol extract $(19.5 \%)$. It was also found that methanol extract showed highest radical scavenging activity $(83.5 \pm 0.3)$ at the concentration of $0.25 \mathrm{mg} / \mathrm{ml}$. Phytochemical analysis showed that highest concentration of total phenol and total flavonoids were observed in methanol extract. The results revealed that the flower of $K$. henryi could be exploited as potential antioxidant additives. 